\title{
Influência da autoavaliação para melhoria do acesso e da qualidade no quantitativo de procedimentos dos Centros de Especialidades Odontológicas
}

\author{
Influence of self-assessment for improving access and quality \\ in the outpatient procedures in the Dental Specialties Centers
}

Leonardo Vilar Filgueiras (https://orcid.org/0000-0002-4771-7004) ${ }^{1}$

Fabiana da Silva Cabreira (https://orcid.org/0000-0002-2606-8754) ${ }^{1}$

Fernando Neves Hugo (https://orcid.org/0000-0003-2222-7719) ${ }^{1}$

Roger Keller Celeste (https://orcid.org/0000-0002-2468-6655) ${ }^{1}$

${ }^{1}$ Faculdade de Odontologia, Universidade Federal do Rio Grande do Sul. R. Ramiro Barcelos 2492, Santa Cecília. 90035-003 Porto Alegre RS Brasil. leovilarf@gmail.com

\begin{abstract}
We studied the influence of Self-Assessment for Improving Access and Quality (AMAQ) in the production of specialized procedures in the of Dental Specialties Centers (DSC). Data were obtained from the 900 DSC submitted to the external evaluation of the Program for Improving Access and Quality (PMAQ-CEO) and the Outpatient Information System (SIA-SUS). The dependent variable was the monthly average of dental procedures (proc/mon) in the DSC performed in three periods: 1) October 2012 to September 2013; 2) October 2013 to September 2014; and 3) October 2014 to September 2015. The main independent variable was the use of AMAQ/CEO, which occurred during the second period. Type of DSC, average number of dentists with a 20-hour workload, strategies to reduce missing appointments, referral support, clinical protocols and coverage of $\mathrm{PHC}$ registered users were used as covariates. Multiple linear regression models were run to estimate adjusted differences. Teams that used AMAQ-CEO had a mean of 515.0 proc/mon, $575.5 \mathrm{proc} / \mathrm{mon}$, and $519.9 \mathrm{proc} / \mathrm{mon}$ in the first, second and third periods, respectively. The DSC that used AMAQ-CEO had a higher mean than those that did not, with an adjusted difference of $32.7 \mathrm{proc} / \mathrm{mon}, 64.7 \mathrm{proc} / \mathrm{mon}$ and $27.7 \mathrm{proc} / \mathrm{mon}$ for the first, second and third periods, respectively. Key words Management, Instrument, Self-assessment
\end{abstract}

Resumo Estudou-se a influência da Autoavaliação para Melhoria do Acesso e Qualidade (AMAQ) na produção de procedimentos especializados nos Centros de Especialidades Odontológicas (CEO). Utilizou-se dados da avaliação externa de 900 CEO do primeiro ciclo do Programa para Melhoria do Acesso e Qualidade (PMAQ/CEO) e Sistema de Informação Ambulatorial (SIA/SUS). A variável dependente foi a média mensal de procedimentos (proc/mês) dos CEO entre os três períodos: 1) outubro de 2012 a setembro de 2013; 2) outubro de 2013 a setembro de 2014; e 3) outubro de 2014 a setembro 2015. A principal variável independente foi o uso da AMAQ/CEO no segundo período. Tipo de CEO, média de cirurgiões-dentistas com carga-horária de 20h, estratégias contra absenteísmo, apoio matricial, protocolos clínicos e cobertura de cadastrados na Estratégia de Saúde Bucal das Família foram covariáveis. Utilizou-se modelos de regressão linear múltipla para análises ajustadas. Equipes que utilizaram a AMAQ-CEO apresentaram média de 515,0 proc/mês, 575,5 proc/mês, e 519,9 proc/mês no primeiro, segundo e terceiro periodos, respectivamente. As equipes que utilizaram AMAQ-CEO tiveram uma maior média de produção do que aquelas que não utilizaram, com diferença ajustada de 32,7 proc/mês, 64,7 proc/mês e 27,7 proc/mês no primeiro, segundo e terceiro períodos, respectivamente.

Palavras-chave Gestão, Instrumento, Autoavaliação 


\section{Introdução}

Os Centros de Especialidades Odontológicas tem sido a estratégia da Política Nacional de Saúde Bucal para garantir a atenção secundária, ofertando procedimentos de maior complexidade. Tais serviços devem se constituir em centros de referências para as unidades de Atenção Primária integrados ao processo de planejamento loco-regional ofertando, minimamente, as especialidades já conhecidas: periodontia, endodontia, cirurgia, estomatologia e pacientes portadores de necessidades especiais ${ }^{1,2}$. Em função do papel dos CEO de aumentar o acesso aos serviços odontológicos especializados, faz-se necessária autoavaliação do serviço para melhorar a assistência e sistema de gestão com a definição clara de metas da oferta de procedimentos por especialidade ${ }^{3-5}$.

Processos autoavaliativos em saúde podem ser compreendidos como métodos sistemáticos para medir fenômenos, ou o desempenho, comparar o resultado obtido com os critérios estabelecidos e fazer uma análise crítica, considerandose a magnitude da diferença além de considerar os próprios atores corresponsáveis pelo processo do cuidado ${ }^{6,7}$. Organizar protocolos a partir desses métodos auxilia uma gestão eficiente com base na indução e monitoramento da produtividade e a fim de garantir acesso e qualidade da atenção em saúde dos usuários do Sistema Único de Saúde ${ }^{8,9}$.

Entender o processo autoavaliativo dos serviços de saúde requer, além da noção clara dos conceitos, identificação dos seus sujeitos e observação de sua capacidade de influenciar as ações de saúde. Nessa lógica, em termos de sua utilidade prática, uma autoavaliação adequada visa reduzir incertezas, melhorar a efetividade das ações para propiciar subsídios na tomada de decisões relevantes $^{10}$. Para a otimização dos procedimentos e serviços, as questões autoavaliativas implicam geralmente na utilização de variáveis estruturais, de processo e de resultado ${ }^{11,12}$, pois estabelecem relações de causalidade imprescindíveis no planejamento em saúde. Aquelas condições que constroem e condicionam o universo de práticas a ser avaliado (recursos financeiros, humanos, físicos, organizacionais), podem favorecer os dados relativos aos processos que definem essas práticas $^{12}$, como os procedimentos clínicos.

O programa de melhoria de acesso e qualidade (PMAQ-CEO) possui vários componentes para avaliação dos CEO, dentre eles a capacidade de atingir metas, a avaliação externa e a autoavaliação $^{1}$, que aconteceram no intervalo temporal próximo. A utilização do instrumento autoavaliativo AMAQ-CEO (Autoavaliação da Melhoria do Acesso e da Qualidade dos Centros de Especialidades Odontológicas) visa alcançar padrões de competências tanto da gestão quanto da equipe ${ }^{1,13,14}$. Para tanto, este instrumento está organizado em duas unidades de avaliação, gestão e equipe, agrupado em quatro dimensões que se desdobram em treze subdimensões. Nesse contexto, a AMAQ-CEO é uma ferramenta desenvolvida para auxiliar os profissionais no seu processo de trabalho e na tomada de decisão possibilitando a definição de padrões de qualidade na organização das ações e nas práticas clínicas ${ }^{13,15}$.

$\mathrm{O}$ acesso e a qualidade do CEO, detalhados a partir dos padrões avaliados, são aspectos desafiadores para as gerências e devem ser abordados à medida que demonstram como as ações e os serviços são praticados nos CEO e como os gerentes, trabalhadores e usuários estão envolvidos $^{14}$. Dessa forma, ter a AMAQ-CEO como uma alternativa para instrumentalizar a avaliação, pode trazer informações importantes para o processo de trabalho das equipes e assim facilitar a mobilização de iniciativas para mudança e aprimoramento dos serviços ${ }^{7,16,17}$. Assim, esse trabalho tem como objetivo verificar se as equipes dos CEO que utilizam a AMAQ como instrumento de autoavaliação alteraram a média de procedimentos mensais realizados.

\section{Metodologia}

Trata-se de um estudo analítico que utilizou dados secundários da produção ambulatorial de todos os Centros de Especialidades Odontológicas (CEO) entre 2012 e 2015, e do questionário da avaliação externa do PMAQ-CEO (Portaria do MS no 261 de fevereiro de 2013). Os dados referentes ao total de procedimentos clínicos foram extraídos do banco de dados do Departamento de Informática do Sistema Ambulatorial do SUS (SIA/DATASUS). Já os dados referentes ao uso do instrumento AMAQ/CEO pelas equipes foram obtidos do questionário da avaliação externa da PMAQ-CEO. Apesar dos Centros de Especialidades Odontológicas executarem outros procedimentos não computáveis para o cumprimento de suas metas, essa pesquisa considerou apenas os procedimentos definidos na Portaria Ministerial $n^{\circ} 1.464 / 2011$, referente ao subgrupo de especialidades.

A avaliação externa do PMAQ-CEO englobou todos os CEO implantados até o período da 
avaliação. Sendo assim, foram elegíveis 930 CEO identificados através do Cadastro Nacional de Estabelecimentos de Saúde (CNES). Desse total, verificou-se que 20 estabelecimentos possuíam o mesmo CNES que Equipes de Saúde da Família (ESF) e que participaram do PMAQ-AB 20 ciclo. Para evitar superestimativas de procedimentos optou-se por removê-los da análise. Quanto à distribuição das médias de procedimentos, constatou-se que havia estabelecimentos com valores extremos de produção (>2.000 procedimentos/ mês) sendo estes excluídos. Logo, foram considerados no estudo 900 estabelecimentos.

Considerando que avaliação externa ocorreu no $1^{\circ}$ semestre de 2014 e que a AMAQ deveria ter sido realizada até 6 meses, estima-se que as equipes que realizaram a AMAQ entre o segundo semestre de 2013 e primeiro semestre de 2014. Assim, foram definidos para este estudo três períodos de um ano cada: primeiro período (outubro de 2012 a setembro de 2013), segundo período (outubro de 2013 a setembro de 2014) e terceiro período (outubro de 2014 a setembro 2015). Para a definição da variável dependente, foram calculadas as médias dos procedimentos realizados pelos CEO em cada um dos três períodos incluindo os procedimentos das especialidades Periodontia, Endodontia e Cirurgia Oral Menor. Os dados foram obtidos a partir dos arquivos disponibilizados pelo SIA/SUS para TABWIN, entre junho e agosto de 2019 por um único pesquisador experiente. Para a tabulação utilizou-se como filtros o CNES, a frequência aprovada de procedimentos para especialidades de Endodontia, Periodontia e Cirurgia a partir do TABWIN versão 4.15.

A principal variável independente foi a combinação de duas perguntas referentes a realização de autoavaliação utilizando a AMAQ-CEO e ao uso dos resultados da autoavaliação na organização do processo de trabalho da equipe. Foram consideradas na categoria "sim" todas as equipes que informaram ter realizado autoavaliação utilizando a AMAQ-CEO e usaram os resultados no planejamento das ações. Por outro lado, na categoria "não" foram incluídas as equipes que relataram não terem utilizado a AMAQ-CEO ou não terem utilizado seus resultados no processo de trabalho. O uso da AMAQ-CEO não foi obrigatório. Entretanto, das equipes que realizaram a autoavaliação apenas 33 não utilizaram esta ferramenta optando por instrumentos desenvolvidos por Secretarias Estaduais ou Municipais de saúde ou outros instrumentos. Considerando que a AMAQ-CEO é disponibilizada em nível nacional para todos os municípios e foi desen- volvida para focar em aspectos de estrutura, processo e resultado da assistência à saúde, optou-se por direcionar este estudo para as equipes que a utilizaram.

Para esse estudo consideramos outras variáveis explicativas organizadas em duas categorias: variáveis de estrutura e processo dos serviços: "tipo de CEO (I, II, III)", "número de cirurgiõesdentistas 20h (Até 5 CD, 5 a 7 CD, 7 a 9 CD e mais de 9 CD)", "estratégias contra absenteísmo (sim, não)", "apoio matricial (sim, não)", "protocolos clínicos por especialidades (sim, não)"; e variáveis contextuais: "cobertura percentual de pessoas cadastrados na Estratégia de Saúde Bucal das Famílias no ano de 2013 (Até 80\%, acima de $80 \%$ )" e "porte populacional (Até 50 mil, 50 a $500 \mathrm{mil}$, acima de $500 \mathrm{mil}$ )”. As variáveis foram coletadas e agrupadas de acordo com as informações presentes no questionário da avaliação externa da PMAQ-CEO, do DATASUS e Instituto Brasileiro de Geografia e Estatística (IBGE).

Os dados extraídos da avaliação externa do PMAQ-CEO foram coletados com instrumento padronizado, testado previamente, respondido pelos gerentes dos CEO e através de observação dos avaliadores in loco. Os avaliadores externos que coletaram os dados foram treinados por Instituições de Ensino e/ou Pesquisa (IEP) sob a coordenação da Coordenação-Geral de Saúde Bucal/Departamento de Atenção Básica. A avaliação externa do PMAQ-CEO foi aprovada pelo Comitê de Ética e Pesquisa da Universidade Federal de Pernambuco (UFPE) com parecer no 740.874 (CAEE: 23458213.0.0000.5208).

Análise estatística descritiva foi utilizada para verificar médias e medidas de dispersão para as variáveis contínuas, frequências relativas e absolutas para as categóricas. Utilizou-se o teste de Kruskal-Wallis no modelo inicial para associações bivariadas, considerando nível de significância de 1\%. Para os modelos de ajuste, foram realizadas regressões lineares múltiplas no segundo e no terceiro período para estimar efeito das variáveis de exposição. A análise estatística foi realizada através do software "R" Studio (versão 1.1.442), 2018.

\section{Resultados}

Na observação dos períodos isoladamente, verificou-se que a produtividade média mensal total no primeiro e terceiro período foi de 530,3 $(\mathrm{DP} \pm 463,2)$ e $540,0(\mathrm{DP} \pm 79,8)$, respectivamente anterior e posterior à avaliação externa da PMA- 
Q-CEO. No segundo período (variável-resposta) onde houve a avaliação externa, essa média foi maior em 542,9 (DP $\pm 421,2)$ procedimentos correspondendo a um aumento de aproximadamente $2,5 \%$ quando comparados aos outros períodos citados.

Já na análise entre os períodos relacionados a cada covariável observada, a média mensal de procedimentos especializados dos CEO tipo III no segundo período foi a maior, mesmo correspondendo ao menor percentual entre os outros tipos de estabelecimentos distribuídos no país em 2013 (10,57\%). Considerando a carga-horária padrão de $20 \mathrm{~h}$ para cada profissional, a média total de procedimentos é maior no segundo período entre $\mathrm{CEO}$ com 5 a $7(488,6 ; \mathrm{DP} \pm 403,4), 7$ a $9(598,6 ; \mathrm{DP} \pm 363,2)$ e acima de 9 cirurgiõesdentistas $(767,2 ; \mathrm{DP} \pm 470,0)$. Equipes que utilizaram a AMAQ-CEO para organização de seus processos de trabalho tiveram um aumento da produção nos três períodos, em comparação as equipes que não fizeram e não utilizaram o instrumento autoavaliativo. No entanto, observouse no segundo período o maior aumento de procedimentos executados $(575,5 ; \mathrm{DP} \pm 432,4)$. Em média, as equipes que possuem estratégias contra absenteísmo, apoio matricial e estabelecem protocolos clínicos produziram mais procedimentos especializados nos três períodos analisados, sendo também no segundo período em que ocorreu a maior diferença entre eles.

Em relação aos dados de cobertura de cadastrados na Estratégia de Saúde da Família, verificou-se que 50,45\% dos CEO estão localizados em municípios com até $80 \%$ de cobertura e possuem maior média mensal de procedimentos executados observado nos três períodos. No entanto, foi no segundo período em que houve maior acréscimo de procedimentos mensais nos dois estratos. CEO em municípios com cobertura acima de $80 \%$ possuíram maior variação da média de procedimentos, entre $7,5 \%$ e $10 \%$, entre os outros dois períodos e quando comparados ao primeiro estrato. Para a variável porte populacional, a maior parte dos CEO está localizado em municípios com até 50 mil habitantes (52,57\%), porém a maior média mensal de procedimentos tanto no segundo período quanto nos outros dois períodos pesquisados, foi em municípios acima de 500 mil habitantes. Todas as associações foram estatisticamente significativas (Tabela 1 ).

Na Tabela 2, o modelo 1 ajustado com todas as covariáveis do estudo explicou $24,7 \%$ da variação da média de procedimentos dos CEO no mesmo período. Nesse modelo verifica-se que as equipes que não utilizaram a AMAQ-CEO no processo de trabalho tiveram -51,55 (IC95\% $-105,9: 2,90)$ procedimentos não executados. No modelo 2, à exceção das variáveis "recebe apoio matricial" e "utiliza protocolos clínicos" que não foram significativas no ajuste do modelo 1 , o número de procedimentos não executados pelo não uso da AMAQ-CEO foi -64,74 (IC95\% -117.5: -11,9). Ambos os modelos apresentaram significância estatística.

Seguindo os mesmos critérios de análise e comparação para verificar o efeito do instrumento autoavaliador antes e após a realização da avaliação externa, as análises brutas do primeiro e terceiro períodos tiveram, respectivamente, -73,04 (IC95\% -135,7: -10,30) e -79,14 (IC95\% 139,4: -18,8) procedimentos a menos apresentando associação estatística. Apesar da redução dos coeficientes e da não significância estatística nos modelos 1 de ajustes, os períodos correspondentes (Tabela 3 e Tabela 4) mantiveram quantitativo negativo de procedimentos especializados não executados ao não uso do instrumento AMAQ-CEO com -23,02 (IC95\% -84,1: 38,1) e $-12,3$ (IC95\% -70,3: 45,7), respectivamente.

\section{Discussão}

O estudo mostrou que a utilização do instrumento AMAQ-CEO tem um efeito no aumento da produtividade especializada pelas equipes nos três períodos analisados, mais especificamente ao segundo período entre setembro de 2013 a outubro de 2014. Os dados apontam que grande parte do aumento da média desses procedimentos especializados nos Centros de Especialidades Odontológicas ocorreu entre os CEO tipo I e III e em equipes com 5 a 7,7 a 9 e mais de 9 cirurgiões-dentistas trabalhando $20 \mathrm{~h}$. A realização e utilização da AMAQ-CEO durante o segundo período estudado sugere um efeito indutor do instrumento na produção especializada, especialmente em municípios mais populosos (acima de $500 \mathrm{mil} / \mathrm{hab}$.) e com maior cobertura das Equipes de Saúde Bucal da Estratégia de Saúde da Família (ESB/ESF).

Sobre a cobertura da atenção básica pelas Equipes de Saúde Bucal da Estratégia de Saúde das Família, os resultados mostram que, para o mesmo período pesquisado, $\mathrm{CEO}$ em municípios com baixa cobertura de ESF/ESB $(\leq 80 \%)$ contribuíram para um maior aumento do número nos procedimentos executados, mostrando que municípios com insuficiente cobertura de ESB/ 
Tabela 1. Descrição de médias de procedimentos mensais dos Centros de Especialidades Odontológicas (CEO), frequências relativas e absolutas por variáveis nos três períodos.

\begin{tabular}{|c|c|c|c|c|}
\hline Variáveis & $\mathbf{N}(\%)^{*}$ & $\begin{array}{c}1^{\circ} \text { Período } \\
\text { (média } \pm \text { DP) }\end{array}$ & $\begin{array}{c}2^{\circ} \text { Período } \\
\text { (média } \pm \text { DP) }\end{array}$ & $\begin{array}{c}\text { 30 Período } \\
\text { (média } \pm \text { DP) }\end{array}$ \\
\hline Produção ambulatorial & $900(100)$ & & & \\
\hline \multicolumn{5}{|l|}{ Tipo de $\mathrm{CEO}^{\star \star}$} \\
\hline I & $345(38,38)$ & $362,1( \pm 366,5)$ & $369,8( \pm 320,4)$ & $359,3( \pm 324,5)$ \\
\hline II & $460(51,63)$ & $595,2( \pm 469,9)$ & $599,6( \pm 410,7)$ & $601,4( \pm 449,3)$ \\
\hline III & $95(10,57)$ & $775,1( \pm 525,2)$ & $848,2( \pm 499,0)$ & $847,5( \pm 503,3)$ \\
\hline \multicolumn{5}{|c|}{ Número de cirurgiões-dentistas ${ }^{\star *}$} \\
\hline Até $5 \mathrm{CD}$ & $287(32,07)$ & $381,8( \pm 360,2)$ & $375,5( \pm 326,4)$ & $380,6( \pm 368,0)$ \\
\hline De 5 a 7 CD & $226(25,25)$ & $458,8( \pm 425,6)$ & $488,6( \pm 403,4)$ & $473,9( \pm 422,8)$ \\
\hline De 7 a 9 & $170(18.99)$ & $594,9( \pm 468,6)$ & $598,6( \pm 363,2)$ & $596,9( \pm 376,2)$ \\
\hline Mais de 9 & $217(23,69)$ & $734,7( \pm 525,1)$ & $767,2( \pm 470,0)$ & $757,6( \pm 494,7)$ \\
\hline \multicolumn{5}{|l|}{ 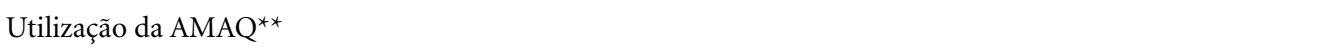 } \\
\hline Sim & $568(63,11)$ & $515,0( \pm 388,5)$ & $575,5( \pm 432,4)$ & $519,9( \pm 373,1)$ \\
\hline Não & $332(36,89)$ & $440,0( \pm 385,9)$ & $472,0( \pm 383,4)$ & $466,9( \pm 393,0)$ \\
\hline \multicolumn{5}{|c|}{ Estratégias para evitar absenteísmo ${ }^{* *}$} \\
\hline Sim & $662(73,56)$ & $510,5( \pm 396,3)$ & $556,3( \pm 400,0)$ & $537,8( \pm 390,7)$ \\
\hline Não & $238(26,44)$ & $423,3( \pm 361,0)$ & $421,9( \pm 328,2)$ & $410,9( \pm 331,8)$ \\
\hline \multicolumn{5}{|l|}{ Apoio matricial } \\
\hline Sim & $459(51,0)$ & $484,1( \pm 365,1)$ & $534,6( \pm 388,9)$ & $518,1( \pm 379,2)$ \\
\hline Não & $441(49,0)$ & $489,4( \pm 412,1)$ & $505,8( \pm 384,1)$ & $489,0( \pm 380,4)$ \\
\hline \multicolumn{5}{|c|}{ Protocolos Clínicos por Especialidades ${ }^{\star *}$} \\
\hline Utiliza & $675(75,0)$ & $520,3( \pm 394,7)$ & $553,3( \pm 390,5)$ & $538,8( \pm 378,4)$ \\
\hline Não utiliza & $225(25,0)$ & $386,1( \pm 352,4)$ & $421,6( \pm 357,6)$ & $400,2( \pm 365,8)$ \\
\hline \multicolumn{5}{|c|}{$\begin{array}{l}\text { Cobertura de Cadastrados na Estratégia de } \\
\text { Saúde da Família }(\%)^{\star *}\end{array}$} \\
\hline Até 80 & $458(50,45)$ & $604,1( \pm 417,2)$ & $628,8( \pm 415,7)$ & $627,1( \pm 405,8)$ \\
\hline$>80$ & $442(49,55)$ & $373,4( \pm 316,5)$ & $413,1( \pm 320,0)$ & $383,6( \pm 305,2)$ \\
\hline \multicolumn{5}{|l|}{ Porte Populacional ${ }^{\star *}$} \\
\hline Até 50 mil & $460(52,57)$ & $348,0( \pm 309,9)$ & $380,4( \pm 300,8)$ & $374,5( \pm 328,0)$ \\
\hline 50 a 500 mil & $222(25,37)$ & $649,1( \pm 472,7)$ & $671,9( \pm 433,6)$ & $680,2( \pm 458,3)$ \\
\hline$>500 \mathrm{mil}$ & $218(22,06)$ & $819,7( \pm 574,1)$ & $779,6( \pm 502,2)$ & $757,8( \pm 532,2)$ \\
\hline
\end{tabular}

Nota: $1^{\circ}$ período de outubro de 2012 a setembro de $2013 ; 2^{\circ}$ período de outubro de 2013 a setembro de $2014 ; 3^{\circ}$ período de outubro de 2014 a setembro de 2015. ${ }^{*}$ Análise descritiva através do teste Kruskal-Wallis; ${ }^{\star *} \mathrm{p}<0,001$ comparado entre as categorias por período.

Fonte: Autores.

ESF apresentam uma maior demanda de serviços no CEO principalmente os de atenção básica, comprometendo a atenção especializada pela incoerência na referência dos casos encaminhados ${ }^{1}$. Alguns estudos esclarecem que o número de CEO em municípios de pequeno porte deve ser analisado com cautela, posto que o funcionamento adequado desses centros depende de pactuações para formação de redes de atenção em saúde bucal previstas nos Planos Diretores de Regionalização ${ }^{3,18}$. A implantação dos CEO em municípios que tenham uma atenção primária consolidada é importante, de modo que a atenção especiali- zada tenha bases para cumprir os princípios da integralidade adequadamente. Ressalta-se que, apesar de ter havido redução da produtividade nos $\mathrm{CEO}$ em municípios com cobertura acima de $80 \%$ no terceiro período, a diferença é insuficiente para verificar se esses municípios melhoraram a oferta de procedimentos da atenção primária, ou apenas reorganizaram o acesso através da operacionalização dos protocolos de referência e contrarreferência.

Em relação ao uso do instrumento AMAQCEO a variação na média dos procedimentos com altos valores de desvio padrão entre o pri- 
Tabela 2. Coeficiente de média mensal de procedimentos no segundoperíodo (out 2013 a set 2014) através de modelo de regressão linear múltipla (IC95\%) para utilização da AMAQ e variáveis relacionadas a avaliação externa da PMAQ realizados nos Centros de Especialidades Odontológicas em 2013.

\begin{tabular}{|c|c|c|c|c|}
\hline Modelos & $\begin{array}{c}\text { Não } \\
\text { usa } \\
\text { AMAQ }\end{array}$ & IC95\% & $\begin{array}{c}\text { p- } \\
\text { valor }\end{array}$ & $\begin{array}{c}\text { R2 } \\
\text { (ajustado) }\end{array}$ \\
\hline Bruto & $-115,09$ & $-171,3:-58,4$ & $<0,001$ & - \\
\hline Modelo $1^{\star}$ & $-51,55$ & $-105: 2,90$ & 0,05 & $24,7 \%$ \\
\hline Modelo $2^{* *}$ & $-64,74$ & -117.5: $-11,9$ & 0,01 & $24,5 \%$ \\
\hline \multicolumn{5}{|c|}{$\begin{array}{l}{ }^{*} \text { Modelo 1: ajustado por tipo de CEO, carga horária/cirurgião- } \\
\text { dentista 20h, estratégias contra absenteísmo, apoio matricial } \\
\text { protocolos clínicos, cobertura de cadastrados na ESF e porte } \\
\text { populacional; }{ }^{* *} \text { Modelo 2: ajustado por tipo de CEO, carga horária/ } \\
\text { cirurgião-dentista } 20 \mathrm{~h} \text {, estratégias contra absenteísmo, cobertura } \\
\text { de cadastrados na ESF e porte populacional. }\end{array}$} \\
\hline
\end{tabular}

Fonte: Autores.

Tabela 3. Coeficiente de média mensal de procedimentos no período (out 2012 a set 2013) através de modelo de regressão linear múltipla (IC95\%) para utilização da AMAQ e variáveis relacionadas a avaliação externa do PMAQ realizados nos Centros de Especialidades Odontológicas em 2013.

\begin{tabular}{lcrrc}
\hline Modelos & $\begin{array}{c}\text { Não } \\
\text { usa } \\
\text { AMAQ }\end{array}$ & IC95\% & $\begin{array}{c}\text { p- } \\
\text { valor }\end{array}$ & $\begin{array}{c}\text { R2 } \\
\text { (ajustado) }\end{array}$ \\
\hline Bruto & $-73,04$ & $-135,7:-10,30$ & $<0,01$ & - \\
Modelo 1* $^{*}$ & $-23,02$ & $-84,1: 38,1$ & 0,46 & $21,1 \%$ \\
Modelo 2** $^{*}-32,70$ & $-91,9: 26,5$ & 0,27 & $21,0 \%$ \\
\hline
\end{tabular}

${ }^{*}$ Modelo 1: ajustado por tipo de CEO, carga horária/cirurgiãodentista 20h, possuir estratégias contra absenteísmo, realiza apoio matricial, protocolos clínicos, cobertura de cadastrados na ESF e porte populacional; ${ }^{*}$ Modelo 2: ajustado por tipo de CEO, carga horária/cirurgião-dentista $20 \mathrm{~h}$, possuir estratégias contra absenteísmo, cobertura de cadastrados na ESF e porte populacional.

Fonte: Autores.

meiro e o segundo período pode estar explicada por dois fatores importantes: 1 ) as equipes que já tinham uma produção maior realizaram a autoavaliação; ou 2) a avaliação externa da PMAQCEO que ocorreu imediatamente após a autoavaliação. Compreendendo que a diferença entre os grupos já existia, mas ficou maior, a autoavaliação pode ter contribuído para esse o aumento no segundo período em comparação à linha de base, assim sua aplicação pode ter efeito indutor nas ações executadas pelas equipes. Já em relação a avaliação externa pela PMAQ-CEO, a mesma não se propõe ao aumento da produção, pois corresponde a uma ferramenta que verifica in loco as
Tabela 4. Coeficiente de média mensal de procedimentos no terceiroperíodo (out 2014 a set 2015) através de modelo de regressão linear múltipla (IC95\%) para utilização da AMAQ e variáveis relacionadas a avaliação externa da PMAQ realizados nos Centros de Especialidades Odontológicas em 2013.

\begin{tabular}{|c|c|c|c|c|}
\hline Modelos & $\begin{array}{c}\text { Não } \\
\text { usa } \\
\text { AMAQ }\end{array}$ & IC95\% & $\begin{array}{c}\text { p- } \\
\text { valor }\end{array}$ & $\begin{array}{c}\text { R2 } \\
\text { (ajustado) }\end{array}$ \\
\hline Bruto & $-79,14$ & $-139,4:-18,8$ & $<0,05$ & - \\
\hline Modelo $1^{\star}$ & $-12,3$ & $-70,3: 45,7$ & 0,67 & $22,6 \%$ \\
\hline Modelo $2^{\star \star}$ & $-27,7$ & $-117.5:-11,9$ & 0,27 & $22,4 \%$ \\
\hline \multicolumn{5}{|c|}{$\begin{array}{l}{ }^{*} \text { Modelo 1: ajustado por tipo de CEO, carga-horária/cirurgião } \\
\text { dentista 20h, estratégias contra absenteísmo, apoio matricial } \\
\text { protocolos clínicos, cobertura de cadastrados na ESF e port } \\
\text { populacional; }{ }^{* *} \text { Modelo } 2 \text { ajustado por tipo de CEO, carga horária } \\
\text { cirurgião-dentista } 20 \mathrm{~h} \text {, estratégias contra absenteísmo, cobertur } \\
\text { de cadastrados na ESF e porte populacional. }\end{array}$} \\
\hline
\end{tabular}

Fonte: Autores.

condições de trabalho e o monitoramento das ações ${ }^{1,14,19}$. No entanto, pode influenciar as equipes na condução dos seus serviços ${ }^{1}$ e de alguma forma ter também contribuído com esse aumento. Fato esse explicado quando percebida a diminuição da média no terceiro período.

A AMAQ-CEO enquanto instrumento autoavaliativo, tem caráter reflexivo e problematizador que contempla diferentes julgamentos entre os atores envolvidos no processo de fazer saúde, sejam usuários, profissionais e os próprios gestores ${ }^{14}$. Dada a sua importância como um instrumento que formula estratégias para melhoria do serviço ${ }^{14}$, o resultado encontrado entre os períodos mostra que o uso da AMAQ-CEO pode colaborar com o processo de trabalho e na organização das equipes de forma positiva ${ }^{19}$.

Nas análises de regressão múltipla referentes ao uso do instrumento autoavaliativo, os modelos brutos mostram uma queda da produtividade nas equipes que não utilizaram a $\mathrm{AMAQ}-\mathrm{CEO}$ no processo de trabalho, especialmente no segundo período (Tabela 2), quando foi feita a avaliação externa do PMAQ-CEO. Sendo a AMAQ-CEO fundamental no PMAQ-CEO para fomentar a autogestão dos problemas, os modelos brutos e ajustados confirmam que existem diferenças estatísticas significantes na não utilização do instrumento e na queda da produtividade dos procedimentos especializados nos CEO. Apesar disso, deve-se considerar a dificuldade de determinar com precisão a latência do efeito da AMAQ-CEO após sua implantação, pois CEO que não utilizaram processos autoavaliativos para o cum- 
primento de metas de produtividade tiveram a produção abaixo do esperado para todos os procedimentos especializados ${ }^{20}$.

Embora os resultados da Tabela 2 expliquem um $\mathrm{R}^{2}$ aproximadamente de $24 \%$, com pouca variação de ajuste nos dois modelos, há uma queda de $-64,74$ de procedimentos no modelo 2 quando removidas as variáveis que não foram associadas no modelo 1 como "receber apoio matricial" e "protocolos clínicos”. Essa diferença na queda da produtividade observada nos $\mathrm{CEO}$ ao não uso da AMAQ em relação a essas variáveis pode ser explicada pela utilização de dispositivos próprios de organização dos processos de trabalho das equipes na elaboração de estratégias de intervenção e melhoria dos seus serviços. Gestão da fila de espera, demanda de profissionais para certa especialidade, tempo e recursos, e acessibilidade do usuário ao atendimento, são elementos que independem de um instrumento autoavaliativo para serem utilizados ${ }^{6,15,21}$. Nesse aspecto, e entendendo que alguns elementos citados anteriormente como a redução da acessibilidade do usuário ao serviço afeta a produção, a utilização de um instrumento autoavaliativo específico pode não ser suficiente quando não se reconhece com antecedência a vulnerabilidade de cada indivíduo e a capacidade de trabalho do serviço ${ }^{22}$ o que pode ser explicado ao observar a maior queda no modelo 2 da Tabela 2. No entanto, sendo essa pesquisa feita com dados secundários sobre o uso de um instrumento autoavaliador na produção, não foi possível verificar quais intervenções foram propostas pelas equipes visando melhorar a produção. Podemos considerar a mesma explicação em relação aos modelos da Tabela 4 dada a observação do efeito pós-realização da AMAQ-CEO ou da avaliação externa nesse período, considerando sua influência na diminuição da queda de procedimentos em relação ao período correspondente à variável-resposta.

A realização e utilização da AMAQ-CEO durante o segundo período estudado sugere um efeito indutor da ferramenta na produção especializada para o período. Tanto na análise bivariada como no modelo ajustado, as equipes que utilizaram a AMAQ-CEO apresentaram maior estimativa média de procedimentos especializados no segundo período e menor estimativa no terceiro período. No modelo ajustado para o segundo período esta diferença foi de 64,7. Sabendo que o CEO tipo I, por exemplo, na soma das especialidades, deve cumprir a meta de 175 procedimentos/mês ${ }^{12}$, esta diferença de 64,7 procedimentos corresponde a $37 \%$ da meta, refor- çando a importância dos processos autoavaliativos na gestão dos serviços. Além disso, outros dois fatores poderiam explicar a variação na média dos procedimentos entre o primeiro e o segundo período. Primeiro, pode-se supor que a diferença de produção entre os estabelecimentos já existia sendo acentuada pela realização e uso da AMAQ-CEO explicando os altos valores de DP encontrados. Segundo, a variação pode ser explicada pela ocorrência da avaliação externa do PMAQ-CEO, que ocorreu imediatamente após a autoavaliação pelas equipes. É possível também que durante os processos avaliativos se observe uma melhora nos serviços avaliados pelo simples fato de estarem sob avaliação. Também se deve considerar a dificuldade de determinar com precisão a latência do efeito tanto da AMAQ-CEO como da avaliação externa, isso é, o tempo entre usar o AMAQ e o momento em que se observa alguma mudança. Entretanto, dada a importância da AMAQ-CEO como uma ferramenta para identificar potencialidades, desafios e promover a autogestão dos problemas e formular estratégias para melhoria do serviço ${ }^{3}$, o resultado encontrado entre os períodos evidencia que o uso da AMAQ-CEO pode contribuir para qualificação do processo de trabalho e na organização das equipes de forma positiva em um curto espaço de tempo.

Como limitação deste estudo, enfatizamos que os dados da pesquisa são referentes apenas aos CEO e não outras unidades de saúde que prestam atenção especializada, um número que é considerado alto $^{23}$. Além disso, dispomos dos dados informados no banco que compõe a avaliação externa da PMAQ-CEO (2013) e não dados específicos das respostas da AMAQ-CEO para averiguar os resultados da autoavaliação de cada CEO. Em adição, os dados de produção dos CEO são usados como parte do processo de certificação, embora os gestores não tenham conhecimento de quais meses são utilizados para tal avaliação. É possível ter havido supernotificação durante o segundo período deste estudo, e assim, o aumento observado pode ser artificialmente fruto de notificações e não um real aumento. Tal hipótese, entretanto, precisa ser confirmada. $\mathrm{O}$ presente estudo é suscetível à qualidade de fontes secundárias, quem são geradas para fins administrativos e não pesquisa. Embora os altos valores de desvio-padrão reflitam a variação de produção entre os $\mathrm{CEO}$, é possível que parte de tal variação seja fruto de erros, assumidos aqui como aleatórios, que tenderiam a reduzir eventuais associações. Todavia, todos as fontes são vinculadas 
a estratégias oficiais do Ministério da Saúde do Brasil melhorias na qualidade do mesmo passa pelo uso amplo de pesquisadores da área.

Por fim, concluímos que os resultados obtidos nessa pesquisa mostram que tanto a AMAQ-CEO quanto a avaliação externa ocorrida no intervalo próximo ao uso do instrumento, podem influenciar na execução das ações e nos processos de trabalho, levando aparentemente a um aumento na produção. O estudo também mostra que o efeito da autoavaliação não é duradouro e deve ser realizado de forma rotineira. Além disso, a presença da avaliação externa contribui para reforçar a expansão das ações buscando melhoria para a Atenção Especializada em Saúde Bucal. Esse estudo pode ser importante para que gestores, profissionais e técnicos em saúde possam tomar iniciativas para o aprimoramento dos serviços especializados no CEO por meio da adoção do instrumento autoavaliativo.

\section{Colaboradores}

LV Filgueiras foi o pesquisador principal responsável pela revista da literatura, coleta e análises dos dados, metodologia, resultados, discussão e concepção final do artigo. FS Cabreira foi copesquisadora e trabalhou na coleta e análise dos dados, resultados e revisão final do artigo. FN Hugo trabalhou na revisão final como coorientador. RK Celeste foi o orientador principal, delineou a metodologia adequada, trabalhou na coleta e análises dos dados, na correção e na revisão final do artigo.

\section{Agradecimentos}

RK Celeste e FN Hugo são bolsistas PQ-CNPQ. 


\section{Referências}

1. Goes PA, Figueiredo N, Neves JC, Silveira FMM, Costa JFR, Pucca Junior GA, Rosales MS. Avaliação da atenção secundária em saúde bucal: uma investigação nos centros de especialidades do Brasil. Cad Saude Publica 2012; 28(Supl. 8):S81-S89.

2. Figueiredo N, Goes PSA. Construção da atenção secundária em saúde bucal: um estudo sobre os Centros de Especialidades Odontológicas em Pernambuco, Brasil. Cad Saude Publica 2009; 25(2):259-267.

3. Chaves SCL, Barros SG, Cruz DN, Figueiredo ACL, Moura BLA, Cangussu MCT. Política Nacional de Saúde Bucal: fatores associados à integralidade do cuidado. Rev Saude Publica 2010; 44(6):1005-1013.

4. Reses MLN. Avaliação da implantação dos Centros de Especialidades Odontológicas em Santa Catarina [dissertação]. Florianópolis: Universidade Federal de Santa Catarina; 2011.

5. Machado FCA, Silva JV, Ferreira MAF. Fatores relacionados ao desempenho de Centros de Especialidades Odontológicas. Cien Saude Colet 2015; 20(4):11491163.

6. Ministério da Saúde (MS). Política Nacional de Atenção Básica. Brasília: MS; 2012.

7. Brasil. Ministério da Saúde (MS). Programa Nacional de Melhoria do Acesso e da Qualidade da Atenção Básica (PMAQ): manual instrutivo. Brasília: MS; 2012.

8. Minayo MCS. Pesquisa Avaliativa por triangulação de métodos. In: Bosi MLM, Mercado FJ, organizadores. Avaliação Qualitativa de Programas de Saúde: Enfoques Emergentes. Petrópolis: Vozes; 2006.

9. Uchimura KY, Bosi MLM. Qualidade e subjetividade na avaliação de programas e serviços de saúde. Cad Saude Publica 2002; 18(6):1561-1569.

10. Hartz ZMA. Institutionalizing the evaluation of health programs and policies in France: cuisine internationals over fast food and sur measure over ready-made. Cad Saude Publica 1999; 15(2):229-260.

11. Donabedian A. The definition of quality: a conceptual exploration. Explorations in quality assessment and monitoring. In: Donabedian A, Arbor A. The definition of quality and approaches to its assessment. Michigan: Health Administration Press; 1988. p. 3-31.

12. Figueiredo N, Silveira FMM, Neves JC, Magalhães BG, Goes PSA. Avaliação de ações da atenção secundária e terciária de saúde bucal. In: Goes PSA, Moysés SJ, organizadores. Planejamento, gestão e avaliação em saúde bucal. São Paulo: Artes Médicas; 2012. p. 195-209.

13. Brasil. Ministério da Saúde (MS). Secretaria de Atenção à Saúde. Departamento de Atenção Primária. Autoavaliação para Melhoria do Acesso e da Qualidade dos Centros de Especialidades Odontológicas - AMAQCEO. Brasília: MS; 2013.

14. Brasil. Ministério da Saúde (MS). Programa Nacional de Melhoria do Acesso e da Qualidade da Atenção Básica. Manual Instrutivo: Saúde Mais Perto de VocêAcesso e Qualidade. Brasília: MS; 2013.
15. Goes PSA, Figueiredo N, Martelli PJL, Luvison IR Werneck MAF, Ribeiro MAB, Araújo ME, Padilha WWN, Lucena EHG. Theoretical and Methodological Aspects of the External Evaluation of the Improvement, Access and Quality of Centers for Dental Specialties Program. Pesqui Bras Odontopediatria Clin Integr 2018; 18(1):34-33.

16. Brasil. Ministério da Saúde (MS). Portaria no 1.654 GM/MS, de 19 de julho de 2011. Dispõe sobre o Programa Nacional de Melhoria do Acesso e da Qualidade da Atenção Básica (PMAQ-AB). Diário Oficial da União; 2015.

17. Linhares PHA, Lira GV, Albuquerque IMN. Avaliação do Programa Nacional de Melhoria do Acesso e da Qualidade da Atenção Básica no estado do Ceará. Saude Debate 2014; 38(Supl.):195-208.

18. Fortuna RFP. A média complexidade no quadro da Política Nacional de Saúde Bucal: uma abordagem do seu processo de desenvolvimento em uma perspectiva regional [tese]. Rio de Janeiro: Universidade do Estado do Rio de Janeiro; 2011.

19. Nunes MF, França MASA, Werneck MF, Goes PS PMAQ - CEO External Evaluation: Revealing Indicators for Planning Services. Pesqui Bras Odontopediatria Clin Integr 2018; 18(1):3178.

20. Lucena EHG. Centros de Especialidades Odontológicas (CEO): Análise a partir dos relatos de planejamento e resultados dos indicadores no Programa Nacional de Melhoria do Acesso e da Qualidade (PMAQ-CEO) [tese]. Brasília: Universidade de Brasília; 2016.

21. Pereira AC, Vazquez FL, Guerra LM, Vitor ES, Ambrosano GMB, Mialhe FL, Meneghim MC. Referência e Contra-Referência na Atenção Secundária em Odontologia em Campinas, SP, Brasil. Cien Saude Colet 2013; 19(1):245-256

22. Zaitter WM, Silva M, Biazevic MGH, Crosato E, Pizzatto E, Michel-Crosato E. Avaliação da acessibilidade do paciente à clínica de especialidades de Endodontia em dois distritos de saúde do município de Curitiba (PR). Rev Sul-Bras Odontol 2009; 6(4):413-420.

23. Celeste RK, Moura FRR, Santos CP, Tovo MF. Analysis of outpatient care in Brazilian municipalities with and without specialized dental clinics, 2010. Cad Saude Publica 2014; 30(3):511-521.

Artigo apresentado em 19/07/2020

Aprovado em 05/11/2020

Versão final apresentada em 07/11/2020

Editores-chefes: Romeu Gomes, Antônio Augusto Moura da Silva 
